\title{
Nécessité d'une surveillance intégrée en matière de santé publique pour aborder les syndémies d'infections transmissibles sexuellement et par le
} sang

\author{
M Murti ${ }^{1,2 \star}$, J Wong $^{3,4}$, M Whelan ${ }^{1}$, C Renda ${ }^{1}$, K Hohenadel $^{1}$, L Macdonald ${ }^{1,2}$, \\ Résumé
}

L'Agence de la santé publique du Canada a récemment formulé une approche nationale visant à aborder les infections transmissibles sexuellement et par le sang (ITSS) dans son nouveau Un cadre d'action pancanadien sur les ITSS : Réduction des répercussions sur la santé des infections transmissibles sexuellement et par le sang au Canada d'ici 2030. Ce cadre préconise une approche intégrée qui met l'accent sur des populations clés qui sont touchées par des pandémies concomitantes (syndémies). Nous soutenons que l'intégration de la surveillance aiderait à caractériser et à comprendre les populations, les régions touchées, les comportements à risque et autres facteurs qui contribuent aux syndémies d'ITSS. La création de systèmes de données jumelées ou couplées qui permettraient d'élaborer des rapports systématiques sur des données intégrées est entravée par des obstacles techniques à l'intégration des réserves de données et par les facteurs liés à l'éthique et à la protection de la vie privée lorsqu'il s'agit de fusionner des données individuelles confidentielles. On peut tirer des leçons de l'expérience de certains territoires de compétence où une meilleure compréhension de la syndémie - grâce à une surveillance intégrée des ITSS - a permis de prendre des décisions plus efficaces et plus efficientes en matière de politiques et de programmes. Parmi les catalyseurs émergents, citons l'élaboration de normes et de lignes directrices sur les données, les investissements dans les ressources afin de surmonter les défis techniques, ainsi que la mobilisation des collectivités pour appuyer une utilisation conforme à l'éthique et non stigmatisante des données intégrées. Les mesures concrètes recommandées dans le cadre donnent l'occasion d'avoir une discussion nationale sur les priorités et les ressources nécessaires pour favoriser la surveillance syndémique des ITSS en vue de préparer des rapports locaux, régionaux et nationaux au Canada.

Citation suggérée: Murti M, Wong J, Whelan M, Renda C, Hohenadel K, Macdonald L, Parry D. Nécessité d'une surveillance intégrée en matière de santé publique pour aborder les syndémies d'infections transmissibles sexuellement et par le sang. Relevé des maladies transmissibles au Canada 2019; 45(2/3):68-72. https://doi. org/10.14745/ccdr.v45i23a03f

Mots clés: surveillance, syndémie, ITS, infections transmissibles par le sang, intégration de données, STBBI

\section{Introduction}

Nous saluons le nouveau Un cadre d'action pancanadien sur les ITSS : Réduction des répercussions sur la santé des infections transmissibles sexuellement et par le sang au Canada d'ici 2030, document qui servira de feuille de route aux autorités sanitaires fédérales, provinciales, territoriales et municipales dans les efforts qu'elles déploient pour réduire l'incidence des ITSS sur la santé au Canada (1). Un des principes directeurs du Cadre consiste à aller au-delà des programmes et des interventions axés sur des infections uniques en utilisant des approches intégrées conçues pour « prendre en compte la complexité et la nature interdépendante des facteurs de risque et des voies de transmission des ITSS »- autrement dit, une approche syndémique.

Merrill Singer, un éminent anthropologue médical, donne la définition suivante : "Une syndémie est l'interaction synergique d'au moins deux problèmes de santé qui contribuent à l'accroissement du fardeau de la santé pour les individus 
ou les communautés» (2). Singer a utilisé l'interaction des infections au virus de l'immunodéficience humaine $(\mathrm{VIH})$ et à la tuberculose pour typifier les interactions entre deux maladies (c.-à-d. la progression plus rapide des symptômes et de la maladie en présence d'infections simultanées comparativement à une infection unique) et les conditions communes d'inégalités en matière de santé (par ex., pauvreté et marginalisation) qui contribuent à leur concentration et à leur propagation (3). Dans un exemple plus récent, une étude menée en Colombie-Britannique caractérisait l'épidémie de syphilis chez les hommes gais, bisexuels et d'autres hommes ayant des relations sexuelles avec des hommes, et son association avec la consommation de drogues, les problèmes de santé mentale et la co-infection par le VIH. En outre, I'association était plus évidente chez les personnes qui présentaient des comorbidités multiples (4). L'adoption d'une approche syndémique rehausse les efforts visant à mesurer et à aborder des facteurs de risque connus pour les maladies uniques en caractérisant leurs effets multiplicatifs et leurs répercussions en présence de plusieurs infections. La syndémie nécessite aussi une mobilisation des communautés et des cadres éthiques afin de s'assurer que les données sont présentées d'une manière qui permette de prévenir une plus grande stigmatisation des groupes vulnérables susceptibles d'être associés à un fardeau de maladie excessif.

Une surveillance de santé publique axée sur une approche intégrée permettant d'éclairer et de prioriser des mesures concrètes favoriserait l'atteinte des objectifs du Cadre.

Actuellement, la plupart des systèmes de surveillance de santé publique mettent l'accent sur des maladies uniques. Ils ne sont pas conçus pour permettre de faire rapport sur des causes multiples et interdépendantes des ITSS, comme des infections simultanées et antérieures, des facteurs de risque communs à plusieurs infections, des déterminants sociaux de la santé et des facteurs environnementaux (par ex., disponibilité des tests de dépistage, lieu du traitement, etc.). La compréhension des répercussions synergiques de ces causes de maladie permettrait de prendre des décisions stratégiques prioritaires en matière opérationnelle, de politiques et de programmes (5). Par exemple, la surveillance du moment et de la prévalence des co-infections transmissibles sexuellement et des facteurs qui influencent le dépistage et le traitement pourrait s'avérer utile dans la planification et l'évaluation des programmes de prophylaxie avant exposition, d'autant plus que ces programmes sont en train d'être renforcés à l'échelle du Canada (6).

Bien qu'un système intégré de surveillance des ITSS soit un objectif louable, créer l'infrastructure de données et les politiques de soutien nécessaire est une tâche imposante. Cet article a pour but de présenter des exemples de territoires de compétence qui réalisent ces travaux et d'explorer les défis et les catalyseurs potentiels liés à l'adoption de systèmes de surveillance intégrée des ITSS.

\section{Exemples d'intégration de la surveillance}

Partout dans le monde, des organismes de santé publique organisent un éventail d'activités pour intégrer leurs systèmes et faire rapport sur les syndémies d'ITSS. À titre d'exemple, citons AtlasPlus, site Web interactif élaboré par les Centers for Disease Control and Prevention (CDC) des États-Unis et conçu pour permettre de visualiser les taux d'ITSS et d'infections à la tuberculose, ainsi que les mesures des déterminants sociaux à l'échelle des États (7). AtlasPlus permet de comparer côte à côte des combinaisons de mesures pour deux infections, ainsi que les déterminants sociaux liés à la région concernée, mais ne comprend pas de données couplées ou jumelées. Cet outil offre deux grands avantages : les données relatives à une maladie unique peuvent être actualisées de façon indépendante, et on peut y superposer $n^{\prime}$ importe quelles mesures de déterminants sociaux ou autres facteurs, pourvu qu'ils soient disponibles à des niveaux géographiques semblables. Bien que les interprétations soient limitées à des associations écologiques, cet outil stimule la production d'hypothèses et une exploration plus poussée.

Les CDC appuient également l'intégration de la surveillance pour le VIH, les infections transmissibles sexuellement (ITS), I'hépatite virale et la tuberculose à l'échelle des États et des municipalités grâce à deux initiatives de financement : I'Outcome Assessment through Systems of Integrated Surveillance (OASIS) lancé en 1998 et le Program Collaboration and Service Integration lancé en 2007 (8,9). Ces deux initiatives fournissent des fonds pour l'élaboration de bases de données et pour permettre aux communautés de pratique établies de renforcer leurs capacités d'analyse et d'utilisation des données intégrées. Le service de santé de New York a créé un ensemble de données jumelées pour une période de dix ans pour des patients atteints d'hépatite virale, de tuberculose, d'ITS et du VIH, ainsi que des statistiques de l'état civil sur les décès. Avec cette cohorte, il a décrit les facteurs de risque communs à plusieurs infections et les taux de mortalité plus élevés associés à des infections multiples (10). Il a plus tard jumelé d'autres maladies et affections non infectieuses - comme la prévalence du diabète - à cet ensemble de données afin d'examiner leurs liens avec les ITSS et la tuberculose (11).

Un autre exemple provient de la direction de la santé publique du comté de Los Angeles, qui a superposé aux cartes de quartier les données sur les grappes de co-infections VIH/ITSS, l'emplacement des sites de dépistage actuels et les niveaux de revenus afin de reconnaître les zones nécessitant un taux élevé de services (12). À l'aide de données jumelées, elle a par la suite élaboré des cartes de profil qui permettent de produire des rapports sur les facteurs sociodémographiques, les comportements, les co-infections et les infections récurrentes chez des patients individuels. Les données sur les sous-groupes démographiques et les caractéristiques des infections aident à mieux cibler les programmes. Elle a aussi classé les districts 
sanitaires d'après ces indicateurs afin de déterminer les zones géographiques prioritaires pour la répartition des ressources pour la prévention et le traitement du VIH et des ITS (13).

Des ordres de gouvernement canadiens ont également utilisé des bases de données couplées existantes pour évaluer les répercussions des co-infections chez des individus. Le 2013 Annual Report on STI and HIV de l'Alberta comprenait une analyse jumelée de la prévalence et du moment de l'apparition des co-infections (avant, en même temps ou après l'infection au $\mathrm{VIH}$ ) chez les patients qui ont reçu un diagnostic de séropositivité entre 2005 et 2013 (14). Ces résultats ont révélé un changement au fil du temps : avant 2007, la majorité des patients avaient reçu un diagnostic d'ITS avant ou en même temps que leur diagnostic d'infection au VIH, alors qu'en 2013, ils étaient deux fois plus susceptibles de recevoir un diagnostic d'ITS après leur diagnostic d'infection au VIH. Bien que la cooccurrence d'infections VIH/ ITS soit bien documentée, ce genre de données de surveillance a aidé à repérer un changement dans les tendances à l'échelle locale au fil du temps. Ce type de renseignement peut servir à orienter les programmes de lutte contre les ITSS et à adapter leurs stratégies de dépistage et de prévention pour les personnes à risque d'être infectées au VIH ou qui ont déjà reçu un diagnostic de séropositivité.

Un exemple de ce virage vers la surveillance intégrée et continue au lieu des études de cohortes uniques est la British Columbia Hepatitis Testers Cohort (BC-HTC). Il s'agit d'une cohorte de recherche axée sur des populations, qui utilise des données de surveillance provinciales sur les ITSS et des données provenant des laboratoires de santé publique qui sont jumelées au registre du cancer de la Colombie-Britannique et aux bases de données sur les ordonnances distribuées et le recours aux soins de santé de la Colombie-Britannique. Cette cohorte a été utilisée pour surveiller une cascade de soins pour l'hépatite $C$ dans des populations clés, y compris les personnes vivant avec le VIH et les utilisateurs de drogues par injection (15). Étant donné que cette cohorte est mise à jour de façon régulière, on s'attend à effectuer des analyses continues afin d'éclairer l'élaboration et l'évaluation des programmes et des politiques visant à lutter contre cette syndémie (16). On applique aussi les algorithmes de jumelage élaborés pour la cohorte BC-HTC dans le but d'améliorer la surveillance systématique effectuée à partir de l'entrepôt de données du BC Centre for Disease Control qui facilite la production des rapports sur les ITSS pour la Colombie-Britannique (17).

\section{Défis et catalyseurs}

Il a fallu de nombreuses années pour créer l'infrastructure nécessaire pour analyser les populations clés, et ce, tant en Colombie-Britannique qu'à New York. À la lumière de ce fait, d'autres territoires de compétence qui n'ont peut-être pas encore entamé ce processus peuvent se demander si les données intégrées sur les ITSS en valent la peine, comparativement à celles qu'on peut obtenir en appliquant les méthodes épidémiologiques traditionnelles à des maladies uniques. Même si la surveillance intégrée jouit d'un certain appui, les aspects ci-dessous peuvent donner lieu à des débats :

- Quelles sont les plus importantes questions auxquelles doit répondre la surveillance intégrée?

- Quelle est l'échelle la plus appropriée (municipale, régionale, nationale) pour poser ces questions?

Les territoires de compétence qui ont déjà l'infrastructure en place devront peut-être relever le défi de travailler avec divers intervenants pour cerner les priorités en matière d'extrants, maintenant qu'il est possible d'effectuer de nombreux types d'analyses. Ici, la disponibilité limitée des ressources peut influencer l'ordre de priorité des activités de surveillance et, en même temps, la surveillance intégrée peut contribuer à une meilleure répartition des ressources.

La surveillance intégrée comporte plusieurs défis. Dans un sondage réalisé en 2007 sur les programmes de lutte contre les ITS financés par les CDC et qui examinait dans quelle mesure la surveillance était intégrée dans ces programmes (18), on a d'abord repéré les obstacles à une intégration accrue, notamment des politiques restrictives en matière de données, surtout les données sur le VIH qui sont extraites d'autres systèmes; des bases de données incompatibles, ce qui compliquait le jumelage des données; le manque de temps et $d$ 'expertise technique pour jumeler et gérer les systèmes de données; ainsi que le manque de ressources financières. À la suite de ce sondage, les CDC ont publié des lignes directrices pour assurer la sécurité et la confidentialité des données sur les ITSS grâce à des normes sur les facteurs techniques et liés à la vie privée dont il faut tenir compte lorsqu'on collige des données sur les ITSS aux échelles municipale, de l'État et nationale (19). Ces lignes directrices établissent aussi des normes de programmes pour les organismes de santé publique subventionnés par les CDC, d'après dix principes directeurs pour la collecte, l'entreposage, la transmission et l'utilisation des données afin d'assurer la sécurité et la confidentialité, et 32 normes abordant les politiques et les responsabilités liées aux programmes, la collecte, I'utilisation, la transmission et la diffusion des données, ainsi que la sécurité physique et électronique des données.

En plus de surmonter les obstacles techniques à la surveillance intégrée, l'engagement des communautés à l'échelon municipal est essentiel pour s'assurer que les renseignements découlant d'une telle intégration n'entraînent une stigmatisation accrue des populations clés. Les progrès réalisés dans le dépistage des ITSS, en particulier les analyses phylogénétiques, offrent non seulement de nouvelles possibilités d'évaluation des syndémies, mais ils soulèvent aussi de nouveaux défis liés à l'éthique et à la protection de la vie privée, étant donné qu'une granularité accrue dans la dynamique de transmission 
peut augmenter la possibilité de stigmatisation de certains individus ou groupes particuliers (20). Une des approches utilisées pour relever ces défis est le "Comité des champions" de I'Ontario HIV Epidemiology and Surveillance Initiative, formé de divers membres de la communauté et de personnes qui ont un vécu précis et pertinent; ces comités examinent tous les renseignements découlant des activités de surveillance du VIH réalisées dans le cadre de l'initiative (21).

\section{Discussion}

Un cadre d'action pancanadien préconise une surveillance qui appuie des approches intégrées pour les populations qui sont particulièrement touchées par les syndémies d'ITSS. Pour atteindre ces objectifs, il serait utile de caractériser et de surveiller les populations touchées par les co-infections d'ITSS et les divers facteurs associés à ces infections. Bien qu'il y ait des défis liés à l'intégration de ces données sur les ITSS et d'importants aspects liés à l'éthique et à la protection de la vie privée dont il faut tenir compte, il existe des exemples de territoires de compétence - dont certains au Canada - qui démontrent qu'il est possible de relever ces défis et illustrent les avantages qui en découlent.

Pour bénéficier des avantages découlant de l'intégration, les organismes qui effectuent la surveillance des ITSS devront relever les défis propres à leurs systèmes d'information et à leurs pratiques connexes de gestion et de dissémination des données. Ils peuvent s'y attaquer par des initiatives de financement, la résolution des problèmes techniques par les communautés de pratique, la production de livres blancs sur les normes de données et l'établissement de cadres pour assurer une utilisation des données couplées conforme à l'éthique.

\section{Conclusion}

Il y a un besoin urgent d'aborder le fardeau croissant des ITSS au Canada. Or, la publication du récent Cadre présente une approche nationale convenue pour y parvenir. En définitive, I'innovation, la collaboration et des investissements seront nécessaires pour faciliter l'intégration de la surveillance des ITSS en vue d'appuyer les objectifs de ce Cadre.

\section{Déclaration des auteurs}

$\mathrm{MM}$ - Conceptualisation, rédaction de la première ébauche, examen et révision

JW - Conceptualisation, rédaction des sections originales, examen et révision

MW - Rédaction - examen et révision

$\mathrm{CR}$ - Rédaction - examen et révision

$\mathrm{KH}$ - Rédaction - examen et révision

LM - Rédaction - examen et révision

DP - Conceptualisation, rédaction des sections originales, examen et révision

\section{Conflit d'intérêts}

Aucun.

\section{Remerciements}

Nous aimerions remercier Lindsay Friedman de nous avoir aidés à rassembler les documents de référence pour cet article.

\section{Financement}

Aucun.

\section{Références}

1. Agence de la santé publique du Canada. Un cadre d'action pancanadien sur les ITSS : Réduction des répercussions sur la santé des infections transmissibles sexuellement et par le sang au Canada d'ici 2030. Ottawa (ON): ASPC; 2018. [Accédé le 30 juil 2018]. https://www.canada.ca/ $\mathrm{fr} /$ sante-publique/services/maladies-infectieuses/santesexuelle-infections-transmissibles-sexuellement/rapportspublications/infections-transmissibles-sexuellement-sangcadre-action.html

2. Singer M. Introducing Syndemics: A Critical Systems Approach to Public and Community Health. Wiley: Jossey-Bass. 2009. 304 p. www.wiley.com/en-us/Introductio $\mathrm{n}+$ to + Syndemics\%3A+A+Critical+Systems+Approach+to+P ublic+and+Community+Health-p-97804704720333Singer M, Bulled N, Ostrach B, Mendenhall E.

3. Syndemics and the biosocial conception of health. Lancet 2017 Mar;389(10072):941-50.DOI PubMed

4. Ferlatte O, Salway T, Samji H, Dove N, Gesink D, Gilbert M, Oliffe JL, Grennan T, Wong J. An application of syndemic theory to identify drivers of the syphilis epidemic among gay, bisexual and other men who have sex with men. Sex Transm Dis 2018 Mar;45(3):163-8. DOI PubMed

5. Weinstock H, Douglas JM Jr, Fenton KA. Toward integration of STD, HIV, TB, and viral hepatitis surveillance. Public Health Rep 2009;124(Suppl 2):5-6. DOI PubMed

6. Traeger MW, Schroeder SE, Wright EJ, Hellard ME, Cornelisse VJ, Doyle JS, Stoové MA. Effects of pre-exposure prophylaxis for the prevention of human immunodeficiency virus infection on sexual risk behavior of men who have sex with men: a systematic review and meta-analysis. Clin Infect Dis 2018 Aug;67(5):676-86. DOI PubMed 
7. Centers for Disease Control and Prevention. NCHHSTP AtlasPlus. Atlanta (GA):CDC;2018. [Accédé le 14 nov 2018]. www.cdc.gov/nchhstp/atlas/index.htm

8. Gaffga NH, Samuel MC, Stenger MR, Stover JA, Newman LM. The OASIS Project: Novel Approaches to Using STD Surveillance Data. Public Health Rep 2009;124 Suppl 2:1-4. DOI PubMed

9. Centers for Disease Control and Prevention. NCHHSTP Program Collaboration and Service Integration (PCSI). Atlanta (GA):US Department of Health and Human Services, Centers for Disease Control and Prevention; 20 June 2018. [Accédé le 14 nov 2018]. www.cdc.gov/nchhstp/ programintegration/default.htm

10. Drobnik A, Pinchoff J, Bushnell G, Ly S, Yuan J, Varma JK, Fuld J. Matching HIV, tuberculosis, viral hepatitis, and sexually transmitted diseases surveillance data, 2000-2010: identification of infectious disease syndemics in New York City. J Public Health Manag Pract 2014 Sep-Oct;20(5):506-12. DOI PubMed

11. Drobnik A, Breskin A, Fuld J, Chan C, Hadler J, Tabaei B, Stennis N, Ahuja S, Wu W, Varma JK. Diabetes among people with tuberculosis, HIV infection, viral hepatitis $B$ and C, and STDs in New York City, 2006-2010. J Public Health Manag Pract 2017 Sep/Oct;23(5):461-7. DOI PubMed

12. Janson M, Hu V, Cheng KJ, Frye D, Kerndt P, Sayles J; Division of HIV and STD Programs. Prioritizing prevention efforts in the areas most impacted in Los Angeles County: a syndemic spatial analysis of HIV and STI burden. Los Angeles (CA): Los Angeles County. Public Health 2012 Mar;22. [cité le 31 juil 2018] http://publichealth.lacounty.gov/dhsp/ Presentations/ScienceSummitSpatial7-12.pdf

13. County of Los Angeles Public Health. 2010-2014 HIV \& STD burden by health district: Guide to the health district profiles. Los Angeles (CA):Los Angeles County Public Health; 18 December 2017. [Accédé le 30 november 2018]. http://publichealth.lacounty.gov/dhsp/Mapping/ Docs/2010-2014_HIV-STD_BurdenByHealthDistrictGuideToHealthDistrictProfiles.pdf

14. Health A. Surveillance and Assessment Branch. Notifiable sexually transmitted infections \& human immunodeficiency virus: 2013 Annual Report. Edmonton (AB): Government of Alberta, February 2015. https://open.alberta.ca/dataset/ b880934d-41ec-4f92-8c6b-6ca34a08bb81/resource/
df2e9225-1614-472e-b5c9-0f34166d4284/download/STIND-Annual-Report-2013.pdf

15. BC Centre for Disease Control. BC Hepatitis Testers Cohort. Vancouver (BC):BCCDC; 2018. [Accédé le 31 juil; 2018]. www.bccdc.ca/our-research/projects/bc-hepatitis-testerscohort

16. McKee G, Butt ZA, Wong S, Salway T, Gilbert M, Wong J, Alvarez M, Chapinal N, Darvishian M, Tyndall MW, Krajden M, Janjua NZ; BC Hepatitis Tests Cohort. Syndemic characterization of HCV, HBV, and HIV co-infections in a large population based cohort study. EClinicalMedicine 2018;4-5(2018):99-108. DOI

17. Wong J, MacDougall L, Guest L, Epstein H, Mussavi Rizi SA, Liu X, Yu A, Kim PH, Frizzell D, Alvarez M, Janjua N, Krajden M, Gilbert M; CPS Epidemiology and Surveillance Team. Integration of surveillance and laboratory data in a sexually transmitted infections and bloodborne infections (STIBB) Data Mart. 27th Canadian Conference on HIV/AIDS Research (CAHR). Vancouver, 26-29 April 2018. Abstract EPHP1.11 https://www.cahr-acrv.ca/wp-content/uploads/2018/04/ CAHR2018-Abstract-Book-with-Errata.pdf

18. Dowell D, Gaffga NH, Weinstock H, Peterman TA. Integration of surveillance for STDs, HIV, Hepatitis, and TB: a survey of U.S. STD control programs. Public Health Rep 2009;124 Suppl 2:31-8. DOI PubMed

19. Centers for Disease Control and Prevention. Data security and confidentiality guidelines for HIV, viral hepatitis, sexually transmitted disease, and tuberculosis programs: standards to facilitate sharing and use of surveillance data for public health action. Atlanta (GA):US Department of Health and Human Services, Centers for Disease Control and Prevention; 2011. [Accédé le 31 juil 2018]. www.cdc.gov/nchhstp/programintegration/docs/ PCSIDataSecurityGuidelines.pdf

20. German D, Grabowski MK, Beyrer C. Enhanced use of phylogenetic data to inform public health approaches to HIV among men who have sex with men. Sex Health 2017 Feb;14(1):89-96. DOI PubMed

21. Ontario HIV Epidemiology and Surveillance Initiative. HIV in Ontario by public health unit: Testing, new diagnoses and care cascade. November 2018. Toronto (ON):OHESI;2018. [Accédé le 29 nov 2018]. www.ohesi.ca/documents/OHESIHIV-by-PHU-2018-11.pdf 